\title{
AN INTERPRETATION FOR MIN-MAX STRUCTURAL DESIGN PROBLEMS INCLUDING A METHOD FOR RELAXING CONSTRAINTS
}

\author{
J. E. TAYLOR \\ Department of Aerospace Engineering, The University of Michigan, Ann Arbor, MI 48109, U.S.A. \\ and \\ Martin P. Bendsøe \\ Mathematical Institute, Technical University of Denmark, DK-2800 Lyngby, Denmark
}

(Received 13 December 1982; in revised form 18 July 1983)

\begin{abstract}
Min-max type problems arise in structural design when the objective is to minimize the maximum value of some local measure of system response, e.g. design to minimize the maximum stress or displacement. A method is described whereby the min-max problem is interpreted as a simple min problem. Governing equations for the adjoint structure are derived directly from the Lagrangian for this min problem by using the generalized multiplier rule on the original state equations. Also certain advantages are demonstrated for a modified form of the min-max problem, a form obtained by introducing a relaxation on the local constraints. The analysis is applied for examples of structural design with stress and displacement criteria, and for the design of an elastic foundation to minimize support pressure.
\end{abstract}

\section{INTRODUCTION}

Practical requircments as constraints in structural optimization problems often lead to mathematical statement in the form of min-max problems. Minimization with respect to the design of the maximum value of stress or displacement over the structure are simple examples of this type of problem. A general method is provided in this paper for the formulation of min-max structural design problems directly via a formal procedure. The method is substantiated on the basis of generalized Kuhn-Tucker theory. The existence of solutions for such design problems is treated as well.

It is common to interpret some of the necessary conditions associated with the cited min-max problems in terms of an adjoint structural analysis problem. The term representing load in the adjoint problem may be singular (e.g. whenever the constraint on local measure of structural response is tight only at points in the domain of the structure). This load is identified as a Dirac function in certain prior treatments of the latter of the examples cited above (e.g. [1, 2]). The support in analysis for this form of the "adjoint load", and for the evaluation of the design derivative as well, is provided in Haug's paper[2]. (A comprehensive and well documented discussion of optimal design relative to displacement constraints is presented in this paper.) The singularity is avoided via the introduction of a " $p$-norm" measure of displacement in the paper by Banichuk et al. [3] on plate design. In related developments presented by Mroz and Mironov[4], where a broader treatment is given for problems of design within mechanical (state) constraints, the "singularity issue" does not arise since only global measures of the constraints are considered.

In the development presented in the next section, the min-max problem is recast as a simple min problem. Governing equations for the adjoint problem are produced here as certain of the necessary conditions associated with the Lagrangian for this min problem.

In a separate part of the development, it is established that with the introduction of a function that comprises a relaxation of the local constraint the entire problem may be analyzed in terms of ordinary functions, i.e. the singularities mentioned above are avoided. Also it is demonstrated that solutions of the relaxed problem converge to the solution of the original one as the relaxation approaches zero.

Two specific example problems are treated in order to demonstrate the method of formulation, and various other applications are discussed as well. 
INTERPRETATION AND ANALYSIS FOR MIN-MAX PROBLEMS

The objective is to establish a variational formulation applicable in a general setting for optimal structural design problems that have the form of min-max problems. For simplicity the development is demonstrated using the particular structural models identified with linearly elastic beams or plates. However similar procedures may be employed for a broad variety of structural design problems.

Function $f(D, w)$ depending on design $D(x)$ and on deflection (state) $w(x)$ and its derivatives is used to represent in general form the criterion for min-max design problems. Thus $f$ expresses some local measure of structural function; examples are the deflection itself $(f=w)$ or a component of stress, or some function of the state of stress. Generally, if the sign of the function is indefinite, then the absolute value of $f$ is used as the criterion. A simple single-criterion design problem has the form

$$
\begin{aligned}
& \min _{D(x)}\left[\max _{x \in D}|f(D, w)|\right] \\
& \text { subject to: } \\
& \text { State equations } \\
& \text { Design constraints. }
\end{aligned}
$$

The set of admissible designs $D$ is defined through the design constraints: $\Omega$ identifies the domain of the structure.

Tiere is in general no assurance that the argument max $[f(D, w)]$ of the above design problem is differentiable. The problem is restated as a simple min problem in terms of a bound, say $\beta$, on the value of the criterion function $f$, i.e.

$$
\operatorname{Min}_{D(x)}(\beta)
$$

Subject to

$$
\begin{array}{rr}
f-\beta \leq 0 & \text { for all } x \in \Omega \\
-\mu f-\beta \leq 0 & \\
a_{D}(w, v)-b(v)=0 & \text { for all } v \in V \\
\text { Design constraints. } &
\end{array}
$$

Within usual restrictions the ordinary variational methods are applicable to this problem. Moreover the solution to problem (1)-(4) may be identified easily with the original min-max problem.

Constant $\mu$ is introduced here to accommodate the slightly broader problem where the upper and lower bounds on $f$ may have different values. In the "virtual work" form of statement for state eqn (3), $a_{D}(\cdot, \cdot)$ is the energy bilinear form associated with structure $D, b$ is the load linear form, and $V \subseteq H^{2}(\Omega)$ represents the space of admissible virtual displacements. The boundary value problem statements corresponding to eqn (3) has the form:

$$
A_{D} w=p, \text { with boundary conditions }
$$

where $A_{D}$ is a linear differential operator depending on design $D$, and $p$ represents load. The admissible designs are defined in terms of resource limitations (volume or weight of material), production constraints, and in terms of constraints ensuring the existence of a proper solution to the optimal design problem; all such constraints are covered by the label "design constraints" in eqn (4).

The procedure by which the original min-max problem is interpreted as indicated in 
(1)-(4) is not novel. Tvergaard [5] uses it in the min-inax (stress) formulation of a fillet design problem, for example. Pedersen and Laursen [6] make use of the same device in subsequent work on computational means to solve discrete design problems. More recently, Miele et al.[7] used the procedure in their treatment of control problems.

Depending on the form of function $f$, singularities may arise out of the constraints (2). The occurrence of singularities, particularly in the multipliers for constraints (2), and the use of a constraint relaxation are discussed in the next section. For the purpose of the development and examples given here, the constraints are taken to be in $L^{2}$.

Within this context, necessary conditions for the problem (1)-(4) may be obtained by formal procedure as stationarity conditions for the augmented functional:

$$
L=\beta+\int_{\Omega}\left\{\eta_{1}(f-\beta)+\eta_{2}(-\mu f-\beta)\right\} \mathrm{d} \Omega-a_{D}(w, v)+b(v)+\psi .
$$

Here $\psi$ represents the constraints of eqn (4). Stationarity of $L$ with respect to $w(x)$ produces the adjoint state (costate) equation. Dems and Mroz (e.g. [8]) in particular have made extensive use of the notion of an adjoint problem in their approach for the variational formulation of structural design. However in their work the adjoint is introduced as part of the statement of the governing functional, rather than being derived from a Lagrangian.

To demonstrate the results for beams as a particular example, suppose that function $f$ in constraint (2) may depend on derivatives of $w(x)$ up to the second, i.e. $f\left(D, w, w^{\prime}, w^{\prime \prime}\right)$, and beam rigidity is represented by $R(D)$ differentiable in $D$. Then

$$
\begin{gathered}
a_{D}(w, v)=\int_{0}^{l} R w^{\prime \prime} v^{n} \mathrm{~d} x \\
b(v)=\int_{0}^{l} p v \mathrm{~d} x \\
A_{D} w=\left(R w^{\prime \prime}\right)^{\prime \prime}
\end{gathered}
$$

and stationarity with respect to variation in $w(x)$ requires

$$
\left(R \bar{w}^{\prime \prime}\right)^{\prime \prime}=\left[\left(\eta_{1}-\eta_{2}\right) \frac{\partial f}{\partial w^{\prime \prime}}\right]^{\prime \prime}-\left[\left(\eta_{1}-\eta_{2}\right) \frac{\partial f}{\partial w^{\prime}}\right]^{\prime}+\left(\eta_{1}-\eta_{2}\right) \frac{\partial f}{\partial w}
$$

Here $\bar{w}$ symbolizes the solution to this (adjoint state) equation. We note the form of dependence of the R.S. of (10), termed the adjoint load, on multipliers $\eta_{1}$ and $\eta_{2}$. Also, for this problem the equation, usually labelled as the "optimality condition", that reflects stationarity of $L$ with respect to design $D$ is:

$$
-\left(\frac{\partial R}{\partial D}\right) w^{\prime \prime} \bar{w}^{\prime \prime}+\left(\eta_{1}-\eta_{2}\right) \frac{\partial f}{\partial D}+\frac{\partial \psi}{\partial D}=0
$$

The first term in this equation is identified with a measure of design sensitivity; the measure itself is termed "mutual specific energy" in the design domain.

Both the original and the adjoint state equations are represented in functional form through the Lagrangian (6a). This feature is emphasized here by rewriting the Lagrangian, using symbols $w$ and $\bar{w}$ for state and adjoint state respectively:

$$
L=\beta+\int_{1}\left\{\eta_{1}(f-\beta)+\eta_{2}(-\mu f-\beta)\right\} \mathrm{d} \Omega-a_{D}(w, \bar{w})+b(\bar{w})+\psi .
$$

To summarize the treatment thus far, an interpretation in the form of a simple min problem has been given for min-max structural design problems, the Lagrangian for this min problem is identified, and the associated adjoint state and optimality condition 
equations have been exhibited. Other implications of the analysis become apparent with a more detailed considerations of the necessary conditions. It is useful in this respect to follow examples of application; the particular design problems treated next serve well enough for this purpose.

\section{EXAMPLES}

Taking the height $h(x)$ of a beam with solid rectangular cross-section as the design variable, the objective in this example problem is to minimize the maximum stress. In this case the stress is proportional to $h w^{\prime \prime}$ so that the design problem is stated:

$$
\min _{h(x)}\left[\max _{x}\left|f=h w^{\prime \prime}\right|\right]
$$

The corresponding min problem is:

$$
\min _{h}(\beta)
$$

Subject to:

$$
\left.\begin{array}{c}
h w^{\prime \prime}-\beta \leq 0 \\
-\mu h w^{\prime \prime}-\beta \leq 0
\end{array}\right\} \begin{gathered}
\int_{0}^{l}\left[\alpha h^{3} w^{\prime \prime} \bar{w}^{\prime \prime}-p \bar{w}\right] \mathrm{d} x=0 \\
h_{\min }-h \leq 0 \\
b \int_{0}^{l} h \mathrm{~d} x-W \leq 0 .
\end{gathered}
$$

The function $\alpha h^{3}$ ( $\alpha=$ constant) equals beam rigidity. Admissible designs are defined in terms of the last two constraints; $W$, the bound on total resource, and $h_{\min }$, the bound on local measure of the design, represent specified non-negative values. Width $b$ of the beam is taken to be constant.

For this problem Lagrangian (6) has the specific form:

$$
\begin{aligned}
L=\beta & +\int_{0}^{l}\left\{\eta_{1}\left(h w^{\prime \prime}-\beta\right)+\eta_{2}\left(-\mu h w^{\prime \prime}-\beta\right)\right. \\
& \left.-\alpha h^{3} w^{\prime \prime} \bar{w}^{\prime \prime}+p \bar{w}+\eta_{3}\left(h_{\min }-h\right)\right\} \mathrm{d} x+\eta_{4}\left[b \int_{0}^{l} h \mathrm{~d} x-W\right] .
\end{aligned}
$$

(Note that the last two terms in this expression were represented in (6) by $\psi$.) Stationarity of $L$ requires

$$
\begin{gathered}
1-\int_{0}^{1}\left(\eta_{1}+\eta_{2}\right) \mathrm{d} x=0 \\
-3 \alpha h^{2} w^{\prime \prime} \bar{w}^{\prime \prime}-\eta_{3}+\eta_{4} b+\left(\eta_{1}-\mu \eta_{2}\right) w^{\prime \prime}=0 \\
-\left(\alpha h^{3} \bar{w}^{\prime \prime}\right)^{\prime \prime}+\left[\left(\eta_{1}-\mu \eta_{2}\right) h\right]^{\prime \prime}=0 \\
\eta_{1}\left(h w^{\prime \prime}-\beta\right)=0 \\
\eta_{2}\left(-\mu h w^{\prime \prime}-\beta\right)=0
\end{gathered}
$$




$$
\begin{gathered}
\text { Structural design problems } \\
\eta_{3}\left(h_{\min }-h\right)=0 \\
\eta_{4}\left(b \int_{0}^{l} h \mathrm{~d} x-W\right)=0 .
\end{gathered}
$$

The solution is governed by these equations together with the original constraints and the (Kuhn-Tucker) conditions $\eta_{i} \geq 0$. The first equation represents a normalization of the multipliers (adjoint load) $\eta_{1}$ and $\eta_{2}$. The second and third equations are simply the specific forms of optimality condition (11) and adjoint state eqn (10) for this example. Note that from eqns (14), (17) and (18), constraints $h w^{\prime \prime}-\beta \leq 0$ and/or $-\mu h w^{\prime \prime}-\beta \leq 0$ are tight at the least somewhere in the domain $(0, l)$. In other words, in the solution for stationarity of $L$, the value of criterion measure $h w^{\prime \prime}$ (or $-\mu h w^{\prime \prime}$ ) at its maximum equals $\beta$. This substantiates the identification of problem (12) with the associated min-max problem, or, in the more general form it serves to identify the problem (1)-(4) with its min-max problem.

From the fourth and fifth equations we have that $\eta_{1}$ and $\eta_{2}$ are orthogonal, $\eta_{1} \cdot \eta_{2}=0$. Additional interpretation of the system provides that $\eta_{4} \neq 0$, whereby the resource constraint is tight. Also, by the switching equation $\eta_{3}\left(h_{\min }-h\right)=0$, the domain $(0, l)$ is covered in intervals with either $h>h_{\min } ; \eta_{3}=0$ (design intervals), or $h=h_{\min } ; \eta_{3} \geq 0$. In the design intervals $\eta_{1} \neq 0, \eta_{2}=0$ and $h w^{\prime \prime}-\beta=0$, or $\eta_{2} \neq 0, \eta_{1}=0$ and $-\mu h w^{\prime \prime}-\beta=0$. Making use of these results the entire system can be reduced by algebraic manipulation to a substantially simpler form.

In fact the solution itself is obtained directly for simple loads; for $p(x)=p_{0} x / l$ as an example, the shape of the optimal beam in design intervals is given by $h(x)=\left[p_{0}\left(x^{3}+c_{1} x+c_{2}\right) / 6 l \mu \beta\right]^{1 / 2}$, where $c_{1}$ and $c_{2}$ are constants. An iterative method is used to determine the boundaries $\left(x_{1}\right.$ and $x_{2}$ in Fig. 1) of design intervals, and the values of $\beta$, integration constants, etc. Details of the solution for a simply supported beam under the cited load and for $\mu=1$ are shown in the figure.

As a second example, we treat the foundation design for a beam on a linearly elastic foundation. The objective is to determine foundation modulus, say $k(x)$, that minimizes the maximum foundation pressure. The magnitude of pressure is given by $|k w|$ ( $w=$ beam deflection), so the design problem is stated as $\min [\max |k w|]$. Note that in this example the criterion function again depends explicitly on the state and the design functions. The "minimize on a bound" form for this problem is:

$$
\min _{k(x)}(\beta)
$$

Subject to:

$$
\begin{aligned}
k w-\beta & \leq 0 \\
-\mu k w-\beta & \leq 0 \\
\int_{0}^{1}\left[R w^{\prime \prime} \bar{w}^{\prime \prime}+(k w-p) \bar{w}\right] \mathrm{d} x & =0 \\
k-k_{\max } & \leq 0 \\
K-\int_{0}^{1} k \mathrm{~d} x & \leq 0 .
\end{aligned}
$$

In contrast to the prior example, here the measure of global resource is bounded from below while the design $k(x)$ is limited locally from above (the system does not admit solutions with $k \leq 0$ ).

The Lagrangian is formed for this problem, and the solution may be established from 


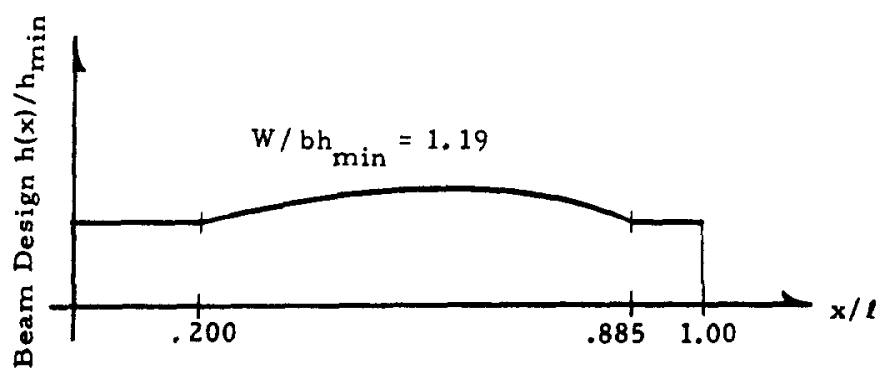

Fig. 1. Optimal design of a simply supported beam to minimize maximum stress.

the associated necessary conditions, in much the same way as was indicated for the first example (for brevity the details are omitted). A solution is sketched in Fig. 2 for the case $p \equiv 0$, hinged supports, and with prescribed, equal-valued displacement of the beam ends into the foundation. As indicated in the figure, the magnitude of foundation pressure is constant in the design intervals $(|k w|=\beta)$ in the optimal solution.

\section{A RELAXED FORM FOR MIN-MAX PROBLEMS}

In problems where the criterion function is directly a measure of state or of its first derivative, the adjoint load (multipliers $\eta_{1}$ and $\eta_{2}$ ) is in general singular. This property is demonstrated for the former case, i.e. $\min _{D}[\max |w(x)|]$, where $w(x)$ represents beam or plate deflection. The Langrangian (6) for the problem is stated as:

$$
L=\beta+\int_{\Omega}\left[\eta_{1}(w-\beta)+\eta_{2}(-\mu w-\beta)\right] \mathrm{d} \Omega-a_{D}(w, \bar{w})+b(\bar{w})+\psi,
$$

and the associated necessary conditions related to multipliers $\eta_{1}$ and $\eta_{2}$ are:

$$
1-\int_{\Omega}\left(\eta_{1}+\eta_{2}\right)=0
$$

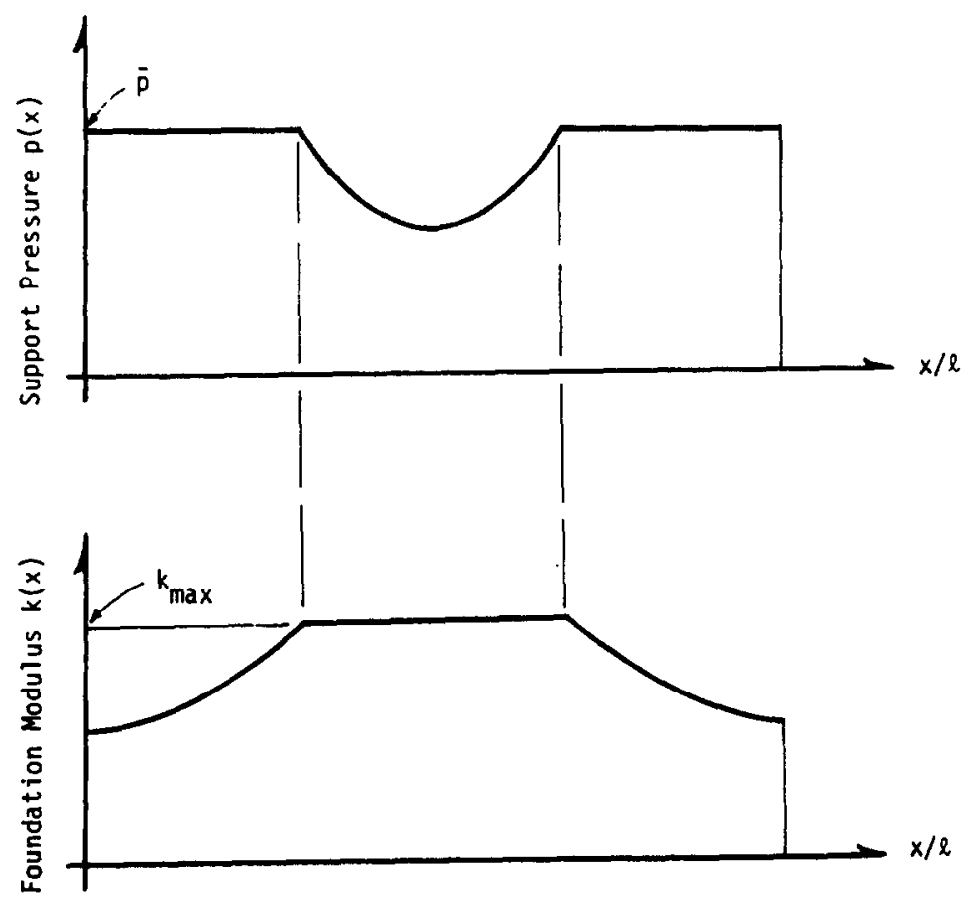

Fig. 2. Optimal design of foundation modulus to minimize maximum foundation pressure. 


$$
\begin{array}{ll}
\eta_{1}(x)=0 \text { if } w(x)<\beta ; & \eta_{2}(x)=0 \text { if }-\mu w(x)<\beta \\
\eta_{1}(x) \geq 0 \text { if } w(x)=\beta ; & \eta_{2}(x) \geq 0 \text { if }-\mu w(x)=\beta .
\end{array}
$$

Typically the deflection function cannot have constant value over any interval of positive measure, whereby the stated conditions (22)-(23) dictate that $\eta_{1}$ and $\eta_{2}$ must be certain linear combinations of Dirac $\delta$-functions. Haug[2] substantiates this result; also in his variational formulation Cinquini[1] identifies the adjoint load as a Dirac-function, but the above normalization (22) is not present in his treatment.)

According to this result, the determination of (adjoint state) $\bar{w}$ requires the solution of a beam or plate boundary value problem with singular loads. As an alternative, by treating this type of problem in a slightly modified form such singularities may be avoided. The modification amounts to a globally-bounded relaxation relative to the original constraint on local measure $f(D, w)$. Thus the prior constraint $|f(D, w)|-\beta \leq 0$ is rewritten in terms of relaxation $\epsilon(x)$ as $|f(D, w)|-(\beta+\epsilon) \leq 0$. In place of a problem statement such as (1)-(4) of the original problem, the relaxed problem is stated as:

$$
\min _{D} \beta
$$

Subject to

$$
\begin{gathered}
\left.\begin{array}{r}
f-(\beta+\epsilon) \leq 0 \\
-\mu f-(\beta+\epsilon) \leq 0
\end{array}\right\} \\
a_{D}(w, v)-b(v)=0 \\
D_{\min }-D \leq 0 \\
\int_{\Omega} D \mathrm{~d} \Omega-W \leq 0 \\
-\epsilon \leq 0 \\
\int \epsilon \mathrm{d} x-E \leq 0
\end{gathered}
$$

with $f$ as before, and $\epsilon \in L^{2}(\Omega) . c, E, D_{\min }$, and $W$ represent specified nonnegative numbers. Thus the relaxed problem corresponds still to minimization with respect to design of the bound value $\beta$, but now with an admissible violation $\epsilon(x)$ (the admissible set is defined by (29)-(30)) of the bound on $f$, where the total measure of violation is not to exceed the value $E$.

The original min-max problem is recovered for $E \rightarrow 0$; this issue is discussed later. The relationships among $w, \epsilon$, and $\beta$ are indicated in the sketch for the case $f=w$.

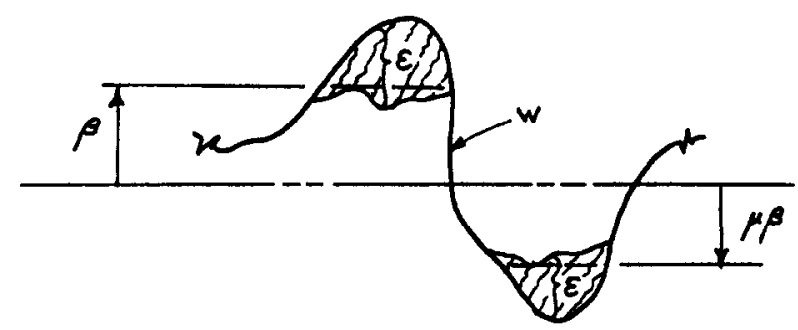

Constraint Relaxation Function $\varepsilon(x)$ 
The optimal relaxation from among admissible functions $\varepsilon(x)$ is associated with equality in one of the constraints (25). In other words, in the solution of the relaxed problem, the difference $f-c$ (or $-\mu f-\epsilon$ ) has constant value equal to the bound $\beta$.

For problems with $E>0$ the load in the adjoint problem is no longer singular; it is verified below that multipliers $\eta_{1}$ and $\eta_{2}$ in fact have constant value wherever they differ from zero. Note also that the introduction of a relaxation regularizes the problem in the sense that constraints (25) are regular even through their counterparts in the original min-max problem may lack regularity (for the case of constraint eqn (2) where $f$ is linear, this issue does not arise).

An augmented functional for the relaxed problem (24)-(30) has the form

$$
\begin{aligned}
L^{*}=\beta & -a_{D}(w, \bar{w})+b(\bar{w})+\int_{\Omega}\left[\eta_{1}(f-\beta-\epsilon)+\eta_{2}(-\mu f-\beta-\epsilon)+\eta_{3}\left(D_{\min }-D\right)\right. \\
& \left.+\eta_{S}(-\epsilon)\right] \mathrm{d} \Omega+\eta_{4}\left[\int_{\Omega} D \mathrm{~d} \Omega-W\right]+\eta_{6}\left[\int_{\Omega} \epsilon \mathrm{d} \Omega-E\right]
\end{aligned}
$$

where the terminology is the same as in (6) except for the additional multipliers $\eta_{s}$ and $\eta_{6}$ associated with the constraints that define admissible $\epsilon(x)$. Stationarity of $L^{*}$ with respect to $\beta, w, \epsilon$ require satisfaction of eqns (14) and (10) as in the original min-max problem, and in addition

$$
-\left(\eta_{1}+\eta_{2}\right)-\eta_{5}+\eta_{6}=0
$$

The solution must also satisfy the conditions:

or

or

$$
\left.\begin{array}{ll}
\eta_{1}=0 & \text { if } f<\beta+\epsilon \\
\eta_{1} \geq 0 & \text { if } f=\beta+c
\end{array}\right\}
$$

or

$$
\left.\begin{array}{lll}
\eta_{2}=0 & \text { if } & -f<\mu \beta+\epsilon \\
\eta_{2} \geq 0 & \text { if } & -f=\mu \beta+\epsilon
\end{array}\right\}
$$

or

$$
\left.\begin{array}{rl}
\left.\begin{array}{rl}
\eta_{5}=0 & \text { if } \quad-\epsilon<0 \\
\eta_{5} \geq 0 & \text { if }-\epsilon=0
\end{array}\right\} \\
\eta_{6}=0 \text { if } \int \epsilon \mathrm{d} \Omega=E<0 \\
\eta_{6} \geq 0 \text { if } \int \epsilon \mathrm{d} \Omega-E=0
\end{array}\right\} .
$$

The prior condition (11) for stationarity of $L$ with respect to design applies as well to the functional $L^{*}$.

The following properties are apparent:

(i) The multipliers $\eta_{1}$ and $\eta_{2}$ are elements of $L^{2}(\Omega)$, since constraint equations (10) and (11) must be in $L^{2}(\Omega)$ when $\epsilon$ is in $L^{2}(\Omega)$. In other words, $\eta_{1}$ and $\eta_{2}$ are elements of the dual of $L^{2}(\Omega)$, i.e. $L^{2}(\Omega)$. Therefore Dirac functions do not appear in the "load" for the adjoint problem in the relaxed or $\epsilon$-min-max problem.

(ii) The constraint (30) $\int \epsilon d \Omega \leq E$ is active, since otherwise eqn (36) requires that $\eta_{6}=0$, which leads in turn to the requirement from eqn (32) that the non-negative functions $\eta_{1}, \eta_{2}, \eta_{5}$ have zero value almost everywhere. The latter condition would violate eqn (14).

(iii) From eqn (35), $\epsilon>0$ implies $\eta_{3}=0$, whereby from (32) $\eta_{1}+\eta_{2}=\eta_{6}$ has constant value for this case. Furthermore, since (from eqns (33) and (34)) $\eta_{1} \cdot \eta_{2}=0$ it follows that when $\epsilon>0$ either

$$
\left(\eta_{1}=\eta_{6} \text { and } \eta_{2}=0\right) \text { or }\left(\eta_{1}=0 \text { and } \eta_{2}=\eta_{6}\right)
$$


Thus either the upper or the lower constraint on $f$ is acctive when $\epsilon$ is non-zero, and the load in the adjoint eqn (10) has constant value. Combining this with the fact that from (ii) $\int \epsilon \mathrm{d} \Omega=E$ leads to the conclusion that the optimal solution takes "maximum advantage" of the possibility for $f$ to exceed the value $\beta$, as afforded by the presence of the relaxation function $\epsilon(x)$.

A reduced form for the set of necessary conditions may be used as a basis to derive an algorithm for numerical computation of the optimal solution. Also, the appearance of state eqn (3) and the adjoint eqn (10) in variational form makes it convenient to apply the finite element method for solving the problem. To have assurance about the stability of the method relative to choice of mesh (mesh-size), the question of existence of the solution to the distributed parameter problem (24)-(30) is important.

It is possible to show existence of solutions to (24)-(30) for all $E \geq 0$, i.e. for both the $\epsilon$-min-max and for the original min-max problems, if the following conditions are met:

-The states $w$ given by the state eqn (3), with designs that satisfy the given production and volume constraints (e.g. (27) and (28)), constitute a weakly compact subset of $H^{2}(\Omega)$.

-The mapping $(w, D) \rightarrow f\left(w, w^{\prime}, w^{\prime \prime}, D\right)$ is continuous and linear as a map into $L^{2}(\Omega)$.

$-\int_{\Omega} \epsilon \mathrm{d} \Omega \leq E$ is replaced by $\|\epsilon\|_{L^{2}} \leq E$.

Note that the latter property does not affect in any significant way the optimality conditions as discussed previously. The first property means that the set of designs must necessarily be $G$-closed, and in most cases the compactness then follows from an imposed lower bound (e.g. (27)) on the designs (Bendsoe [9]). The second property ensures that the inequality constraints (25) can be rewritten as equality constraints for continuous, convex functionals: if $g^{+}$denotes the positive part of a function, i.e.:

$$
g^{+}(x)=\left\{\begin{array}{cc}
0 & \text { if } g(x) \leq 0 \\
g(x) & \text { if } g(x)>0
\end{array}\right.
$$

then the constraints (25) can be rewritten as:

$$
\int_{\Omega}\left[ \pm f\left(w, w^{\prime}, w^{\prime \prime}, D\right)-\beta-\epsilon\right]^{+2} \mathrm{~d} \Omega=0 .
$$

The details of the existence proof are given in Appendix 1.

For example, in the plate design problem with thickness $h$ of the plate as the design variable, the first property is satisfied if (see Bendsoe [9])

$$
\begin{aligned}
& h \in H^{\prime}(\Omega), \int_{\Omega} h \mathrm{~d} \Omega \leq W, h \geq h_{\min }>0 \text { a.e. } \\
& \|h\|_{H^{1}}=\left(\int_{\Omega}\left(h^{2}+(\operatorname{grad} h)^{2}\right) \mathrm{d} \Omega\right)^{1 / 2} \leq M
\end{aligned}
$$

i.e. we impose a constraint on the slope of the design-variable. Niordson[10] and Litvinov[11] introduced this kind of constraint in their treatment of plate design problems.

Let us finally note that if existence of solutions to the problem (24)-(30) is guaranteed by the satisfaction of the above conditions, then when the bound $E$ on the "violationfunction" $\epsilon$ goes to zero, the solutions of the $\epsilon$-min-max problem (with $\mu=1$ ) will converge to a solution of the original min-max problem. (A proof of this is provided in Appendix 2.) In this way the problem (24)-(30) for small $E$ is identified as an approximation to the original min-max problem, and $E$ can be thought of as a perturbation factor.

\section{EXAMPLE PROBLEM}

The main features of the relaxed formulation are to be illustrated via the treatment of an example problem. We consider the design of a beam to minimize maximum displacement, i.e. the case $f=w$ cited at the beginning of this section. Suppose beam cross-section $A(x)$ is the design variable, and for this example the beam rigidity $R(x)$ is taken to be 
proportional to $A^{k}$, i.e. $R(x)=r A^{k}(x)$. Then the state equation is given by $\int_{0}^{l}\left(r A^{k} w^{\prime \prime} v^{\prime \prime}-p v\right) \mathrm{d} x=0$, and $L^{*}$ is obtained from (31) with $f, D$, and $D_{\min }$ replaced by $w, A$, and $A_{\min }$. To proceed toward a specific solution, for a cantilevered beam supported at $x=0$ under uniform load, $w(x)$ increases monotonically with $x$. Thus there is a value, say $x_{0}$, such that

$$
c(x)=0 \text { for } 0 \leq x \leq x_{0} ; \quad c(x)>0 \text { for } x_{0}<x \leq l .
$$

The corresponding load $\eta_{1}$ in the adjoint problem is (the notation for multipliers $\eta_{1}$ through $\eta_{6}$ is the same as in eqn (31); also since $w(x) \geq 0, \eta_{2}=0$ ):

$$
\eta_{1}(x)=\left\{\begin{array}{cc}
0 & \text { for } 0 \leq x \leq x_{0} \\
\frac{1}{l-x_{0}} & \text { for } x_{0}<x \leq l
\end{array}\right.
$$

Note that $\eta_{1}$ has constant value wherever it differs from zero. Without the constraint relaxation $\epsilon(x)$, the adjoint load is given by the Dirac $\delta$-function at $x=L$.

Integrating the state and adjoint equations leads to

$$
\begin{gathered}
A^{k} w^{n}=F(x)=\frac{1}{2} P_{0}(l-x)^{2} \\
A^{k} \bar{w}^{\prime \prime}=G(x)= \begin{cases}\frac{1}{2}\left(l+x_{0}\right)-x & \text { for } 0 \leq x \leq x_{0} \\
(l-x)^{2} / 2\left(l-x_{0}\right) & \text { for } x_{0}<x \leq l\end{cases}
\end{gathered}
$$

The design itself may be expressed as

$$
A(x)= \begin{cases}A_{\min } & \text { for } x_{1} \leq x \leq l \\ k+1 \sqrt{k F(x) G(x) / \eta_{6}} & \text { for } 0 \leq x \leq x_{1}\end{cases}
$$

where the value $x_{1}$ is obtained from

$$
k F\left(x_{1}\right) G\left(x_{1}\right)=\eta_{6} A_{\min }^{k+1}
$$

which is the optimality condition (27) evaluated for the point $x_{1}$. Taking specific values $l=1, k=1, A_{\min }=1, p_{0}=2$ and $\tilde{W}=5.15$, the complete design is given by $x_{0}=1 / 2$, $x_{1}=3 / 4$ and:

$$
A(x)= \begin{cases}1 & \text { for } 3 / 4 \leq x \leq 1 \\ 16(1-x)^{2} & \text { for } 1 / 2 \leq x \leq 3 / 4 \\ 16(1-x) \sqrt{3 / 4-x} & \text { for } 0 \leq x \leq 1 / 2\end{cases}
$$

This design is pictured in Fig. 3.

\section{OTHER APPLICATIONS}

The convenience afforded by the transformation of min-max design problems to simple min problems and by the relaxation of constraints, as demonstrated on the beam examples, might be realized in the context of various other problems in mechanics and optimal design. Two other types of design problems are discussed briefly in this section, the problem of design for elastic bodies in contact, and the formulation for optimal remodel design.

The objective to minimize relative to design the maximum value of contact pressure may be taken as the basis for optimization in contact problems (see, e.g. [12]). The case where the purpose is to design the initial gap, say $g_{0}(x)$, between the bodies is used as a particular 


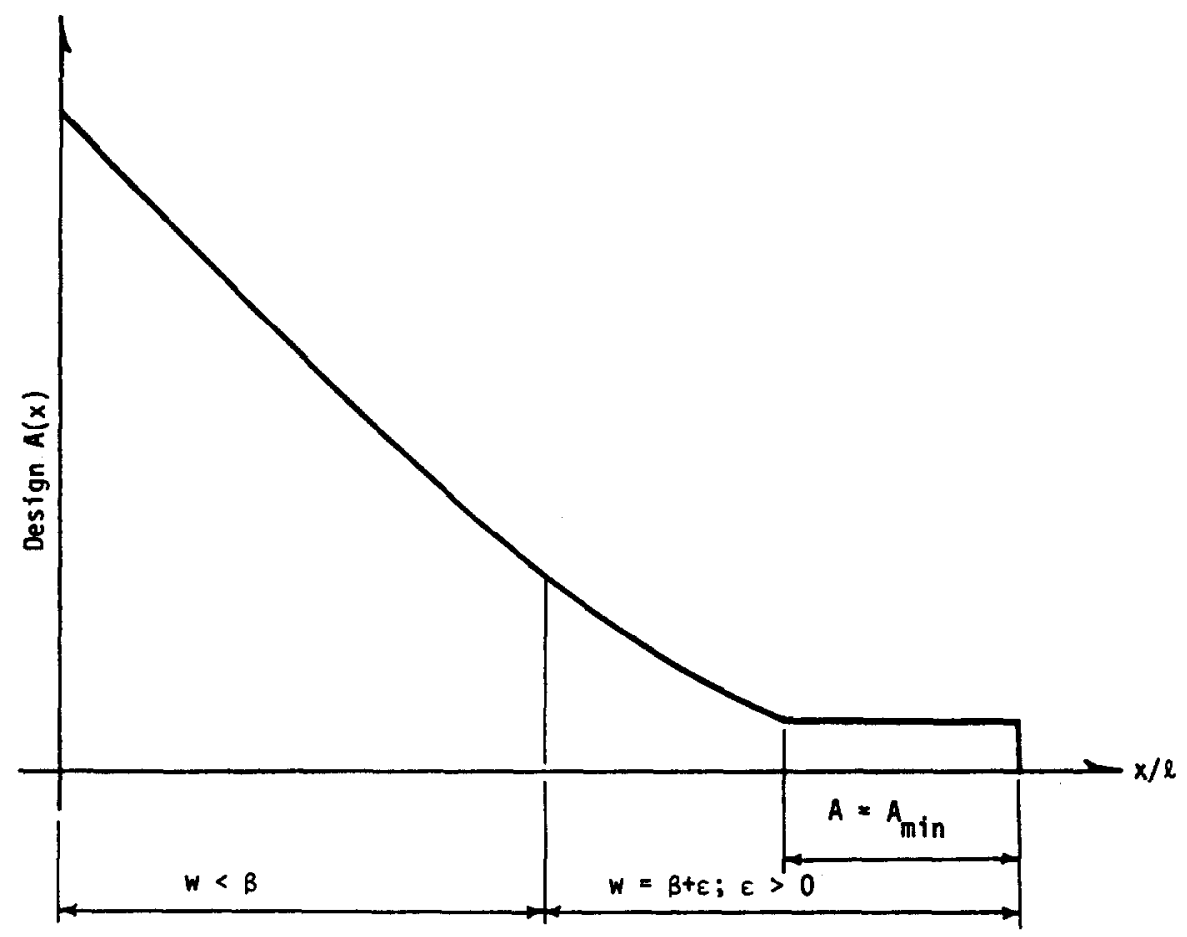

Fig. 3. Optimal design of a cantilevered beam within a relaxed constraint on displacement.

example. Thus the problem is stated as:

$$
\min _{B_{0} \in \Gamma}\left(\max _{x \in X} p(x)\right)
$$

Subject to:

$$
\begin{aligned}
& \text { "State equations" } \\
& -g_{0}(x) \leq 0 \\
& \int g_{0} \mathrm{~d} x-G \leq 0 .
\end{aligned}
$$

The corresponding min problem, stated in terms of bound $\bar{p}$ on pressure, has the form:

$$
\min _{8_{0} \in \Gamma} \bar{p}
$$

Subject to:

$$
\begin{aligned}
& \text { "State equations" } \\
& \begin{array}{l}
p-\bar{p} \leq 0 \\
-g_{0} \leq 0 \\
\int g_{0} \mathrm{~d} x-G \leq 0 .
\end{array}
\end{aligned}
$$


Pressure $p(x)$ is of course a function of state. (In its present usage, the term "state equations" is intended to reflect the usual constraints for contact problems, as well as the governing field equations and boundary conditions. In the procedure of [12], for example, the gap constraint

$$
g(x)=g_{0}(x)-\bar{u}(x)-\bar{u}(x) \geq 0
$$

was appended to the potential energy for the two bodies; then the associated multiplier turns out to be contact pressure. Here $\bar{u}^{\prime}$ and $\bar{u}^{I I}$ represent outer normal displacement of the two bodies evaluated along the contact boundary interval.)

The necessary conditions for the min problem reproduce results obtained earlier, but with the clear advantage that the Lagrangian for the problem in this form directly reflects the original design objective. Also, the introduction of a constraint relaxation may be useful in the analysis for contact problems, e.g. the presence of a relaxation $\epsilon(x) \neq 0$ in the constraint gap $g(x)$ assures that ordinary functions will suffice to express the contact pressure.

The formulation of optimal remodel design represents a quite different example application. The name refers to the type of problem where the purpose is to predict the design for optimum modification of a given structure, rather than the overall optimal design. For the problem in its general form[13], the solution may represent a combination of reinforcement (added material) over some parts, and lightening (removed material) over other parts of the domain of the structure.

The purpose of discussing the problem here is to point out that the general remodel problem can be represented quite simply with the use of a relaxation in the local constraint on design. The problem is stated:

$$
\min _{\mathbb{u}(x)} \beta
$$

Subject to:

$$
\begin{array}{r}
\text { "State equations" } \\
f-\beta \leq 0 \\
-\mu f-\beta \leq 0 \\
-(d+\epsilon) \leq 0 \\
-\epsilon \leq 0 \\
E-\int_{\Omega} \epsilon \mathrm{d} x \leq 0 \\
\int_{\Omega}(d+\epsilon) \mathrm{d} x-D \leq 0
\end{array}
$$

Here $\mathrm{d}(x)$ represents a remodel design, and $\epsilon(x)$ may be interpreted as a relaxation of $-\mathrm{d}(x) \leq 0$. For the case $D>0$ and $E=0$, the problem statement corresponds to "reinforcement only" modifications. On the other hand, a value $E>0$ is the global measure of material removed. It may be verified from the necessary conditions for this problem statement that if both $D>0$ and $E>0$, the design modification $\mathrm{d}(x)$ must be negative over some intervals of the design domain and positive over others, i.e. $\mathrm{d}(x)$ represents a general remodel.

\section{CONCLUSION}

A variational formulation has been demonstrated for the sort of min-max problems that are typical in structural optimization. This formulation, expressed as a simple min problem 
with appropriate constraints, makes it posible to treat the min-max design problem directly, without the need to interpret constraint functions in terms of a global measure.

Other considerations motivate the treatment of such problems in modified form, a form obtained by introducing a relaxation into certain of the constraints. It is shown in particular that in cases where singularities might otherwise appear in the adjoint problem, such singularities are not present in the modified form.

Acknowiledgements-Work leading to the developments reported in this paper was supported in part (JET) by the National Science Foundation. U.S.A., and in part (MPB) under grant number 16-3019.M-745 from the Danish Council for Scientific and Industrial Rescarch.

\section{REFERENCES}

1. C. Cinquini, Optimal elastic design for prescribed maximum deflection. J. Structural Mech. 7(1), 21-34 (1979).

2. E. J. Haug, A unified theory of optimization of structures with displacement and compliance constraints. J. Structural Mech. $9(4), 415-437$ (1981).

3. N. Banichuk, V. M. Karelishvili and A. Mironov, Optimization problems with local performance criteria in the theory of plate bending. Mekh. Tuerd. Tela 13(1), 116-122 (1978).

4. Z. Mrøz and A. Mironov, Optimal design for global mechanical constraints. Arch. Mech. (Warsaw), 32(4), 505-516 (1980).

5. V. Tvergaard, On the optimum shape of a fillet. Proc. IUTAM Symp., Warsaw 1973, Optimization in Structural Design (Edited by A. Sawczuk and Z. Mroz), pp. 181-195. Springer-Verlag, Berlin (1975).

6. P. Pedersen and C. L. Laursen, Design for minimum stress concentration by finite elements and linear programming. J. Structural Mech. 10(4), 375-392 (1982-83).

7. A. Mielc, B. P. Mohanty, P. Venkataraman and Y. M. Kuo, Numerical solution of minimax problems of optimal control-1. J. Opt. Theory Applic. 38(1), 97-109 (1982).

8. K. Dems and Z. Mróz, Variational approach by means of adjoint systems to structural optimization and sensitivity analysis with boundary variation. Int. J. Solids Structures (in press).

9. M. P. Bendsoe, On obtaining a solution to optimization problems for solid, elastic plates by restriction of the design space. J. Structural Mech., to appear.

10. F. Niordson, Optimal design of plates with a constraint on the slope of the thickness function. Int. J. Solids Siruchures (to appear).

II. V. G. Litvinov, Optimal control of the natural frequency of a plate for variable thickness. USSR Comput. Maths. Moth. Phys. 19, 70-86 (1980).

12. R. L. Benedict and J. E. Taylor, Optimal design for elastic bodies in contact. Proc. of NATO-ASI, Iowa City, Jowa, USA, 20 May-4 June 1980. Optimization of Distributed Parameter Structures (Edited E. J. Haug and J. Cea), Vol. 2, pp. 1563-1599. Sijthoff and Noordhofi, Netherlands (1981).

13. N. Olhoff and J. E. Taylor, On optimal structural remodeling. J. Opt. Theory Applic. 27, 571-582 (1979).

14. L. McLinden, An application of Ekeland's theorem to minimax problems. Nonlinear Anal. Appl. $6(2)$, 189-196 (1982).

\section{APPENDIX}

\section{Existence proof}

Problems $(24)-(30)$ of the section on analytical development can be formulated as follows; with $\Phi(w, \epsilon, \beta)=\beta$

$$
\underset{-, \varepsilon, \rho}{\operatorname{minimize}} \Phi
$$

subject to

$$
\begin{gathered}
F(w, \epsilon, \beta)=\left(\int(f(w)-\epsilon-\beta)^{+2} d \Omega\right)^{1 / 2}=0 \\
G(w, \epsilon, \beta)=\left(\int(-f(w)-\epsilon-\alpha \beta)^{+2} d \Omega\right)^{1 / 2}=0 \\
\epsilon \geq 0,\|\epsilon\|_{\mathcal{L}} \leq E \\
w \in Y
\end{gathered}
$$

where $\Phi: X \rightarrow \Re, X=H^{2}(\Omega) \times L^{2}(\Omega) \times \Omega$ and $Y$ is the set of states (responses) corresponding to the admissible set of designs. We will assume that:

$$
\begin{aligned}
& -w \rightarrow f(w) \text { is linear and continuous as a map from } H^{2}(\Omega) \text { to } L^{2}(\Omega) \text {. } \\
& -Y \text { is weakly compact in } H^{2}(\Omega) \text {. }
\end{aligned}
$$

First note that

$$
Z=\left\{c \in L^{2}(\Omega) \mid c \geq 0,\|c\|_{L} \leq E\right\}
$$


is convex, closed and bounded and thus weakly compact in the reflexive space $L^{2}(\Omega)$. As $Y$ by assumption is weakly compact we thus know that for a minimizing sequence of designs and the corresponding minimizing sequence $\left(w_{n}, \epsilon_{n}, \beta_{n}\right)$ for $\phi$, there exists a weak limit $(w, \epsilon, \beta) \in Y \times Z \times\left\{\right.$ for a subsequence of $\left(w_{n}, \epsilon_{n}, \beta_{n}\right)$. As $w \in Y$ we have an admissible design and as $\epsilon \in Z$ we have (1.4) satisfied.

Since $\Phi$ is weakly continuous, existence for the problem $(1.1)-(1.5)$ may be proved simply by showing that if elements in a sequence $\left(w_{n}, c_{n}, \beta_{n}\right)$ in $X$ satisfies (1.2) and (1.3), then a weak limil $(w, c, \beta)$ also satisfies $(1.2)$ and (1.3). In other words, it is necessary to show that

$$
\text { F ' }(\{0\}) \text { and } G{ }^{\prime}(\{0\})
$$

are weakly closed sets of $X$. The assumptions on $f$ assures that $F$ and $G$ are convex and continuous, so that $F^{-1}(\{0\})$ and $G^{-1}(\{0\})$ are convex and closed sets of $X$ and thus weakly closed.

Continuity and convexity of $F$ and $G$ may be shown as follows. From the fact that $F$ (and likewise for $G$ ) is the combination $A_{4} \circ A_{3} \circ A_{2} \circ A_{1}$ of the following continuous maps:

$$
\begin{array}{lll}
A_{1}:(w, C, \beta) \rightarrow\left(f\left(W^{\prime}\right), C, \beta\right) & \text { as a map } & H^{2} \times L^{2} \times G \rightarrow \rightarrow\left(L^{2}\right)^{3} \\
A_{2}:(w, C, \beta) \rightarrow w-C-\beta & \text { as a map } & \left(L^{2}\right)^{3} \rightarrow L^{2} \\
A_{3}: w \rightarrow w^{+} & \text {as a map } & L^{2} \rightarrow L^{2} \\
A_{4}: w \rightarrow\|w\|_{L^{2}} & \text { as a map } & L^{2} \rightarrow G C
\end{array}
$$

we get that $F$ is a continuous map. The convexity of $G$ (and likewise for $F$ ) is seen from the following calculations:

$$
\begin{aligned}
& G\left(\lambda\left(w_{1}, \epsilon_{1}, \beta_{1}\right)+(1-\lambda)\left(w_{2}, \epsilon_{2}, \beta_{2}\right)\right) \\
& =\left\|\left(-f\left(\lambda w_{1}+(1-\lambda) w_{2}\right)-\lambda \epsilon_{1}-(1-\lambda) \epsilon_{2}-\lambda \beta_{1}-(1-\lambda) \beta_{2}\right)^{+}\right\|_{2} \\
& \leq\left\|\lambda\left(-f\left(w_{1}\right)-\epsilon_{1}-\beta_{1}\right)^{+}+(1-\lambda)\left(-f\left(w_{2}\right)-\epsilon_{2}-\beta_{2}\right)^{+}\right\|_{2} \\
& \leq \lambda G\left(w_{1}, \epsilon_{1}, \beta_{1}\right)+(1-\lambda) G\left(w_{2}, \epsilon_{2}, \beta_{2}\right)
\end{aligned}
$$

for $0 \leq \lambda \leq 1$.

\section{APPENDIX 2}

Convergence of the modified problem for $\mathrm{E} \rightarrow 0$

The notation and assumptions of this appendix are the same as in Appendix 1; we thus consider the problem

$$
\operatorname{minimize}(w, c, \beta)=\beta
$$

subject to

$$
\begin{gathered}
(w, c, \beta) \in F^{-1}(\{0\}) n G^{-1}(\{0\}) \\
\epsilon \leq 0,\|\epsilon\|_{L^{2}} \leq E \\
w \in Y
\end{gathered}
$$

where $\Phi: X \rightarrow \mathscr{R}, X=H^{2}(\Omega) \times L^{2}(\Omega) \times \mathscr{R}$ is weakly continuous, and the constraint set in $X$ given by (2.2)-(2.4) is weakly compact.

Now let $\left(E_{n}\right)$ be a sequence of numbers so that $E_{n} \rightarrow 0$ for $n \rightarrow \propto$, and let $\left(w_{n}, c_{n}, \beta_{n}\right)$ be the corresponding sequence of solutions to the problem (2.1)-(2.4). Thus $c_{n} \rightarrow 0$ in $L^{2}$ by (2.3) and also $c_{n} \rightarrow 0$ weakly in $L^{2}$, and as $Y$ is weakly compact there exists in $Y$ a weak limit $w$ for a subsequence of $\left(w_{n}\right)$. Finally, the sequence $\beta_{n}$ is bounded by the minimizing value for the problem $(2.1)-(2.4)$ with $E=0$, so $\beta_{n}$ converges to a number $\beta$. In all, we have that $(w, 0, \beta)$ is a weak limit for a subsequence of $\left(w_{n}, c_{n}, \beta_{n}\right)$, and because $F^{-1}(\{0\})$ and $G^{-1}(\{0\})$ are weakly closed, $(w, 0, \beta)$ satisfies the constraint $(2.2) ;(w, 0, \beta)$ is thus a solution to $(2.1)-(2.4)$ with $E=0$.

We have thus shown that the solutions to the c-minimax problem $(2.1)-(2.4)$ for $E \rightarrow 0$ converges to a solution to the original min-max problem (for the given assumptions) in the sense that the minimum values converge and the resulting designs converge in the sense of homogenization, i.e. the corresponding states converge weakly. For design spaces chosen as in eqn (38), which restricts the gradient of the designs, we also have weak convergence of the designs themselves (see [9]).

Convergence properties and existence theorems have been studied in greater detail for more general c-min-max problems by McLinden[14]. 